\title{
Performance of Eigenvalue-Based Spectrum Sensing with Approximate Eigenvalue Estimation Methods
}

\author{
Dayan A. Guimarães, Guilherme P. Aquino, Rafaela R. Santos, and Ana C. V. dos Reis
}

\begin{abstract}
This article presents a performance and computational run-time analysis of cooperative spectrum sensing techniques based on the eigenvalues of the received signal sample covariance matrix, under three methods for approximate eigenvalue estimation: the Cholesky iterations algorithm, the Gershgorin theorem with transformed covariance matrix, and the conventional Gershgorin theorem. Widely used eigenvaluebased test statistics are addressed: the generalized likelihood ratio test, the ratio between the maximum and the minimum eigenvalues, and the Roy's largest root test. Simulation results show that the first two eigenvalue estimation methods can yield comparable performances, whereas the latter may operate satisfactorily only in situations of high signal-to-noise ratios. It is also demonstrated that the test statistics are not equally sensitive to eigenvalue estimation errors. The Cholesky iterations algorithm is attractive in terms of run-time and performance for all spectrum sensing techniques, while the simple Gershgoring method may be attractive for the Roy's largest root test.
\end{abstract}

Index Terms - Cognitive Radio, Cooperative Spectrum Sensing, Eigenvalue Estimation.

\section{INTRODUCTION}

The current fixed radio-frequency allocation policy for wireless communications, combined with the high growth in the number of different systems and the demand for new telecommunications services, culminated in the congestion and scarcity of the radio-frequency spectrum. However, some studies have shown that, actually, there is a high underutilization of the frequency bands already allocated to the primary networks (those that own a paid license to operate in such bands) [1]. In this context, the cognitive radio (CR) technology [2] has been considered as a promising solution to the problem of congestion and spectrum scarcity, bringing, among other benefits, the possibility of a shared use of spectrum between secondary networks (those having no paid license) and the primary ones.

Among the various functionalities of a $\mathrm{CR}$, it is the spectrum sensing [3], [4] that allows for the detection of vacant bands, also called spectrum holes or whitespaces. Spectrum sensing can be performed by a single $\mathrm{CR}$, or by multiple CRs in a cooperative basis. The cooperative spectrum sensing (CSS)

D. A. Guimarães, G. P. Aquino, R. R. Santos and A. C. V. dos Reis are with the National Institute of Telecommunications (Instituto Nacional de Telecomunicações - Inatel), Santa Rita do Sapucaí, MG, Brazil. E-mails: dayan@inatel.br, guilhermeaquino@inatel.br, rafaelasantos@get.inatel.br, anareis@get.inatel.br.

This work was supported in part by CNPq under Grant 308365/2017-8, and by RNP, with resources from MCTIC, Grant No. 01250.075413/2018 04, under the Radiocommunication Reference Center (Centro de Referência em Radiocomunicações - CRR) project of the National Institute of Telecommunications (Instituto Nacional de Telecomunicações - Inatel), Brazil. doi: $10.14209 /$ jcis. 2019.25 has the advantage of providing high reliability to the primary signal detection process, thanks to the spatial diversity gain produced by the CRs localized in different geographic positions. As a consequence, multipath fading, shadowing and the hidden terminal problem can be efficiently mitigated [3].

The CSS can be distributed or centralized. In the first approach, the information about the state of the sensed band is exchanged among the CRs in cooperation, and the global decision is made according to the cooperation rule, for example by means of consensus. In centralized CSS, the CRs' decisions, the received signal samples or some quantity derived from these samples are sent via low-bandwidth control channels to a central element of the secondary network, called fusion center (FC), where the global decision about the occupation state of the sensed band is made. When the CRs' decisions are the spectrum sensing information transmitted to the FC, a decision-fusion centralized CSS takes place. If the received samples or other related data are the information transmitted to the FC, we have a data-fusion centralized CSS.

Many spectrum sensing techniques have already been developed, for instance the energy detection, the matched filter detection, the cyclostationary feature detection and the eigenvalue-based detection [3]-[5]. Among these techniques, a great interest has been directed to the eigenvalue-based CSS. In this CSS technique, the test statistics are based on the eigenvalues of the received signal covariance matrix.

The main advantages of eigenvalue-based detection are the high statistical power and the needlessness of knowledge of the sensed signal characteristics. In some eigenvalue-based strategies, the thermal noise power at the receiver is not needed as well [6], meaning that such strategies are considered blind.

The most known eigenvalue-based detection test statistics are the generalized likelihood ratio test (GLRT), the maximum-minimum eigenvalue detection (MMED), also known as eigenvalue ratio detection (ERD), and the maximum eigenvalue detection (MED), also known as Roy's largest root test (RLRT) [6], [7]. These test statistics are typically computed at the FC, under the data-fusion centralized CSS approach.

The GLRT and the MMED do not need knowledge about the primary signal characteristics and about the noise that impairs the sensed signal; therefore, they are blind. The MED is considered semi-blind since it does not need to know the primary signal characteristics, but it needs the thermal noise variance information to form the test statistic. 


\section{A. Motivation}

In any eigenvalue-based detection technique, it is reasonable to believe that the spectrum sensing performance are influenced by the accuracy of the eigenvalue estimates. However, the literature always considers perfect eigenvalue estimates when reporting results on the performance of such techniques. To the best of the authors' knowledge, no publication has focused on proposing approximate solution methods for computing eigenvalues in the context of spectrum sensing. Consequently, no analysis of the influence of eigenvalue estimation errors on the performance of eigenvalue-based spectrum sensing, when it is implemented from different test statistics, has been reported so far. It is added to this context the fact that the computational complexity of traditional numerical methods for eigenvalue computation is high, motivating the search for simpler ones that, additionally, deliver just the enough precision to reach target spectrum sensing performances. Hence, it is of relevant importance to address these research gaps.

\section{B. Contributions and Structure of the Article}

Aiming at filling the above-mentioned gaps, this paper presents a performance analysis of the eigenvalue-based cooperative spectrum sensing under the GLRT, the MMED and the MED test statistics, applying three methods of approximate eigenvalue estimation: the Cholesky iterations algorithm, the Gershgorin theorem with transformed covariance matrix, and the conventional Gershgorin theorem. The performance results achieved with these techniques are compared with those obtained with an almost exact eigenvalue computation using the Matlab eig function ${ }^{1}$, which makes use of well-established, reliable and numerically stable methods. A computational runtime measurement of each method is also provided, establishing a basis for choosing the one that yields the best trade-off between performance and computational burden.

The analysis reported herein can be used for choosing and implementing eigenvalue-based spectrum sensing techniques in real hardware, since it provides the basic building blocks of suitable algorithms for solving the eigenvalue problem in a way that is appropriately fitted to the spectrum sensing context.

The remainder of the paper is organized as follows: Section II presents the system model and the test statistics chosen for analysis, and formulates the problem to be tackled. The approximate eigenvalue estimation methods are described in Section III. Section IV is devoted to the numerical results and interpretations. The conclusions are drawn in Section V.

\section{System Model And PROBlem Description}

The spectrum sensing process can be seen as a binary hypothesis test for which are defined the null hypothesis $\mathcal{H}_{0}$ (primary transmitter disabled) and the alternative hypothesis $\mathcal{H}_{1}$ (primary transmitter enabled). The spectrum sensing performance is commonly measured by means of the probability

\footnotetext{
${ }^{1}$ The Matlab eig function also makes use of iterative computation methods and, thus, it is not exact by definition. Nonetheless, its accuracy in terms of numerical Linear Algebra is high, allowing it to be considered a very precise solution in the context of eigenvalue-based spectrum sensing. Thus, hereafter the results provided by the Matlab eig function are referred to as exact ones.
}

of detection, $P_{\mathrm{d}}=\operatorname{Pr}\left\{T>\gamma \mid \mathcal{H}_{1}\right\}$, and the probability of false alarm, $P_{\mathrm{fa}}=\operatorname{Pr}\left\{T>\gamma \mid \mathcal{H}_{0}\right\}$, where $\operatorname{Pr}\{\cdot\}$ denotes the probability of the underlying event, $T$ is the test statistic associated to the detection technique (GLRT, MMED and MED in this paper) and $\gamma$ is the decision threshold. $P_{\mathrm{d}}$ is the probability of making a global decision in favor of the presence of the primary signal in the sensed band, given that the band is indeed occupied. $P_{\mathrm{fa}}$ is the probability of deciding in favor of the presence of the primary signal given that, in fact, the sensed band is vacant. A high $P_{\mathrm{d}}$ is desirable to reduce the interference caused by the secondary network in the primary network due to missed detections. On the other hand, $P_{\mathrm{fa}}$ needs to be small for more opportunistic transmissions in the secondary network. However, if $P_{\mathrm{d}}$ is increased, so does $P_{\mathrm{fa}}$.

These conflicting probabilities are typically expressed by means of a ROC (receiver operating characteristic) curve, which establishes a trade-off between them as the decision threshold is varied. A performance metric that is also often used is the area under the ROC curve, the AUC. The worst performance corresponds to a ROC curve with $P_{\mathrm{d}}=P_{\mathrm{fa}}$, which yields AUC $=0.5$. In this case it is said that the operating points are on the no-discrimination line. The best performance corresponds to a ROC curve passing through the points $P_{\mathrm{d}}=1$ and $P_{\mathrm{fa}}=0$, yielding $\mathrm{AUC}=1$. Thus, the AUC of a given detector lies in between 0.5 and 1 .

\section{A. Eigenvalue-based Cooperative Spectrum Sensing Model}

Assume that there are $m$ sensors (CRs) or one sensor with $m$ antennas, each one collecting $n$ samples of the received signal from $p$ primary transmitters during a given sensing interval. These samples are transmitted to the FC, where they are arranged in the matrix $\mathbf{Y} \in \mathbb{C}^{m \times n}$, which, under the hypotheses $\mathcal{H}_{0}$ and $\mathcal{H}_{1}$, is given by

$$
\mathbf{Y}=\left\{\begin{array}{ll}
\mathbf{V} & : \mathcal{H}_{0} \\
\mathbf{H X}+\mathbf{V} & : \mathcal{H}_{1}
\end{array},\right.
$$

where the matrix $\mathbf{X} \in \mathbb{C}^{p \times n}$ contains the samples of the transmitted signals, assumed to be zero-mean unit-variance Gaussian random variables that represent the envelope fluctuations of typical digital-modulated and filtered signals [6]. The matrix $\mathbf{V} \in \mathbb{C}^{m \times n}$ contains the samples of the additive white Gaussian noise (AWGN) with zero mean and variance $\sigma^{2}$. The channel matrix $\mathbf{H} \in \mathbb{C}^{m \times n}$ is formed elements $\left\{h_{i j}\right\}$ that represent the channel gains between the $j$-th primary transmitter and the $i$-th $\mathrm{CR}$, for $j=1,2, \ldots, p$, and $i=1,2, \ldots, m$. These elements can be complex random variables to model time-varying fading channels, or can be constants to represent pure AWGN channels.

Without loss of generality, the average power gain of the channel is assumed to be unitary, that is, $\mathbb{E}\left\{h_{i j}^{2}\right\}=1$, where $\mathbb{E}$ is the expectation operator. Hence, the average signal-to-noise ratio (SNR) over all CRs is simply $\mathrm{SNR}=1 / \sigma^{2}$.

In data-fusion eigenvalue-based spectrum sensing [5], [6], the spectrum holes are detected by means of test statistics formed from the eigenvalues of the received signal sample covariance matrix, which is computed at the FC as

$$
\mathbf{R}_{\mathbf{Y}}=\frac{1}{n} \mathbf{Y} \mathbf{Y}^{\dagger}
$$


where $\dagger$ denotes the conjugate transpose operation. Matrix $\mathbf{R}_{Y}$ is Hermitian positive definite [8] and has a real main diagonal, meaning that all its eigenvalues are positive and real $^{2}$.

Subsequently to the computation of $\mathbf{R}_{\mathrm{Y}}$, its ordered eigenvalues $\left\{\lambda_{1} \geq \lambda_{1} \geq \cdots \geq \lambda_{m}\right\}$ are estimated by the $\mathrm{FC}$ and the test statistics GLRT, MMED and MED are respectively calculated according to [6]

$$
\begin{gathered}
T_{\mathrm{GLRT}}=\frac{\lambda_{1}}{\frac{1}{m} \sum_{i=1}^{m} \lambda_{i}}, \\
T_{\mathrm{MMED}}=\frac{\lambda_{1}}{\lambda_{m}}, \\
T_{\mathrm{MED}}=\frac{\lambda_{1}}{\sigma^{2}} .
\end{gathered}
$$

Notice that, as already mentioned, the GLRT and the MMED are completely blind, whereas the MED is semi-blind due to the need of the noise variance information.

\section{B. Problem Description}

From (3), (4) and (5), it is clear that the computation of the eigenvalue-based test statistics in practice subsumes the use of sufficiently accurate estimates of the eigenvalues of the sample covariance matrix $\mathbf{R}_{\mathrm{Y}}$ defined in (2). From the Linear Algebra theory, these eigenvalues are the roots of the characteristic equation [10]

$$
\operatorname{det}\left(\mathbf{R}_{\mathbf{Y}}-\lambda \mathbf{I}\right)=0,
$$

where $\operatorname{det}(\cdot)$ is the determinant of the underlying matrix and I is the identity matrix of order $m$. Although Equation (6) has great importance in theoretical analysis, it is not used to compute the eigenvalues of a matrix in practice [11]. Efficient numerical methods are commonly used instead, which are part of the broad family of eigenvalue problem solution algorithms. All these algorithms are iterative in nature, and what is sought in their implementations is a high convergence rate coupled with accurate eigenvalue (and eigenvector) estimates [11].

For Hermitian matrices, which is the case of $\mathbf{R}_{Y}$, algorithms that are mostly used are the QR algorithm (for matrices with order $m \leq 25$ ) [12], and the Lanczos algorithm (for moderated-to-high values of $m$, less than a few thousands) [11], [13]. The Jacobi algorithm is another well-known alternative; it has been considered in microchip implementations [14] in the context of eigenvalue-based spectrum sensing [15].

In terms of computational tools, it is worth mentioning the EISPACK routine and its successor LAPACK [16], both present in the eig and other Matlab functions, in which the QR algorithm is embedded, along with other ones specific to each function and to the particular structure of the input matrix. These and other related routines have been considered standard when dealing with numerical methods for solving the eigenvalue problem [11]. In this paper, the eigenvalues estimated by the Matlab eig function (see footnote 1) are used

\footnotetext{
${ }^{2}$ A Hermitian matrix $\mathbf{A}$ of order $m \times m$ is a complex square matrix that is equal to its own conjugate transpose, i.e. $\mathbf{A}=\mathbf{A}^{\dagger}$. A Hermitian matrix $\mathbf{A}$ is positive definite if the scalar $\mathbf{z}^{\dagger} \mathbf{A z}$ is strictly positive for every non-zero vector $\mathbf{z}$ of dimension $m$. A positive definite matrix has positive eigenvalues [9, Appendix C].
}

as a benchmark for evaluating the accuracy of other methods, which are described in the next section.

\section{ApProximate Eigenvalue Estimation Methods}

It is noteworthy that the implementation of any of the widespread algorithms discussed in the literature for estimating the eigenvalues of $\mathbf{R}_{Y}$ will produce accurate enough outcomes, such that the spectrum sensing performance degradation resultant from any residual estimation errors (from the point of view of numerical Linear Algebra) will be practically insignificant. In this section we describe methods for approximate computation of the eigenvalues of $\mathbf{R}_{Y}$, aiming at a reduced computational burden with respect to the more accurate ones. Subsequently, in Section IV, the impact of the approximations on the probabilities of detection and false alarm of the GLRT, the MMED and the MED are assessed.

\section{A. Cholesky Iterations}

The Cholesky iterations method [17] for Hermitian matrices is presented in Algorithm 1, where $\operatorname{diag}(\mathbf{J})$ returns a vector formed by the elements of the main diagonal of $\mathbf{J}$, and $\operatorname{chol}(\mathbf{J})$ denotes the Cholesky decomposition (or Cholesky factorization) [10] of $\mathbf{J}$. The Cholesky decomposition of a positive definite Hermitian matrix is given by the product between a lower triangular ${ }^{3}$ matrix $\mathbf{L}$ and its conjugate transpose. In the Algorithm 1, it means that $\mathbf{J}=\mathbf{L} \mathbf{L}^{\dagger}$.

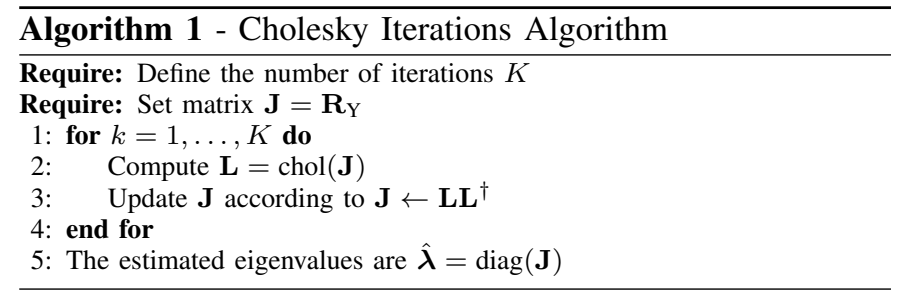

The operation $\operatorname{chol}(\mathbf{J})$ returns the matrix $\mathbf{L}$, and in this paper it has been computed by the Matlab function chol. This function assumes that $\mathbf{J}$ is a positive definite matrix with real main diagonal, as is the case of $\mathbf{R}_{\mathrm{Y}}$. However, due to numerical rounding errors it is possible that the main diagonal of $\mathbf{R}_{Y}$ have almost-zero imaginary residual values that can hinder the use the chol function. To solve this potential problem it is suggested eliminating the residues using the command $J=J-\operatorname{diag}(\operatorname{diag}(J))+\operatorname{real}(\operatorname{diag}(\operatorname{diag}(J)))$ in the Matlab, soon after the matrix $\mathbf{J}$ is created in Algorithm 1.

It is informative to mention that the Cholesky iterations algorithm can produce very accurate eigenvalue estimates if the number of iterations $K$ is large enough. Thus, here $K$ is made the control variable that will determine the tradeoff between the computational burden and the degree of accuracy of the method for comparisons with the built-in Matlab function eig.

\footnotetext{
${ }^{3} \mathrm{~A}$ square matrix is called lower triangular if the entries above the main diagonal are zero.
} 


\section{B. Conventional Gershgorin Theorem}

In the Gershgorin (sometimes appearing as "Gersgorin" and "Gerschgorin" in the literature) theorem method, here denoted as conventional Gershgorin method, the received signal covariance matrix directly provides the information needed to estimate the eigenvalues, without any further processing.

Let $r_{i j}$ denote the element on the $i$-th row and $j$-th column of $\mathbf{R}_{\mathrm{Y}}$, for $i, j=1,2, \ldots, m$. Define the sum of the absolute values of the off-diagonal elements in the $i$-th row of $\mathbf{R}_{Y}$ as $R_{i}=\sum_{j \neq i}\left|r_{i j}\right|$. Define $D\left(r_{i i}, R_{i}\right)$ as disks centralized in $r_{i i}$ with radius $R_{i}$, which are named Gershgorin disks (or Gershgorin circles). The Gershgorin circles theorem [12, p. 82] states that the eigenvalues of $\mathbf{R}_{\mathrm{Y}}$ are located in the union of the disks $D\left(r_{i i}, R_{i}\right)$ in the complex plane. Therefore, the values of $r_{i i}$ can be interpreted as the eigenvalue estimates of $\mathbf{R}_{\mathrm{Y}}$, which will be located on the positive side of the real axis due to the fact that $\mathbf{R}_{Y}$ is positive definite. The accuracy of the $i$-th eigenvalue estimate is associated with the corresponding radius $R_{i}$. This means that the lower the absolute values of the off-diagonal elements of $\mathbf{R}_{\mathbf{Y}}$, the more accurate are the eigenvalue estimates.

\section{Gershgorin Theorem with Transformed Matrix}

Due to possibly large radius of the Gershgorin disks and very close apart centers, the conventional Gershgorin method may produce rather inaccurate eigenvalue estimates. The Gershgorin method with transformed covariance matrix comes as an improvement. It applies a unitary transformation to $\mathbf{R}_{\mathrm{Y}}$, which maintains its eigenvalues, but reduces the radii of the Gershgorin discs and, consequently, improves the accuracy of the eigenvalue estimates [18].

Briefly, in the transformation process of the covariance matrix, $\mathbf{R}_{Y}$ is firstly partitioned according to

$$
\mathbf{R}_{Y}=\left[\begin{array}{cccc}
r_{11} & r_{12} & \cdots & r_{1 m} \\
r_{21} & r_{22} & \cdots & r_{2 m} \\
\vdots & \vdots & \ddots & \vdots \\
r_{m 1} & r_{m 2} & \cdots & r_{m m}
\end{array}\right]=\left[\begin{array}{cc}
\mathbf{R}_{1} & \mathbf{r} \\
\mathbf{r}^{\dagger} & r_{m m}
\end{array}\right]
$$

where $\mathbf{R}_{1}$ is the main sub-matrix of $\mathbf{R}_{\mathrm{Y}}$, of order $(m-1) \times(m-1)$, obtained by removing the last row and the last column of $\mathbf{R}_{Y}$. The vector $\mathbf{r}$, of length $(m-1)$, is the last column of $\mathbf{R}_{\mathbf{Y}}$ without the last element $r_{m m}$. The sub-matrix $\mathbf{R}_{1}$ can be factorized according to

$$
\mathbf{R}_{1}=\mathbf{U}_{1} \mathbf{D}_{1} \mathbf{U}_{1}^{\dagger}
$$

where $\mathbf{U}_{1}$ is a unitary matrix of order $(m-1) \times(m-1)$, composed of the eigenvectors of $\mathbf{R}_{1}$, and $\mathbf{D}_{1}$ is a diagonal matrix with the same order, whose main diagonal is formed by the eigenvalues of $\mathbf{R}_{1}$.

From $\mathbf{U}_{1}$, the unitary transformation matrix is constructed according to

$$
\mathbf{U}=\left[\begin{array}{ll}
\mathbf{U}_{1} & \mathbf{0} \\
\mathbf{0}^{\mathrm{T}} & 1
\end{array}\right],
$$

where $\mathbf{0}$ is the all-zero vector of length $(m-1)$. Finally, the transformed covariance matrix is given by

$$
\mathbf{S}=\mathbf{U}^{\dagger} \mathbf{R}_{\mathbf{Y}} \mathbf{U}=\left[\begin{array}{cc}
\mathbf{D}_{1} & \mathbf{U}_{1}^{\dagger} \mathbf{r} \\
\mathbf{r}^{\dagger} \mathbf{U}_{1} & r_{m m}
\end{array}\right],
$$

which in expanded form is given by

$$
\mathbf{S}=\left[\begin{array}{cccc}
\hat{\lambda_{1}} & 0 & \cdots & \rho_{1} \\
0 & \hat{\lambda_{2}} & \cdots & \rho_{2} \\
\vdots & \vdots & \ddots & \vdots \\
\rho_{1}^{*} & \rho_{2}^{*} & \cdots & \hat{\lambda_{m}}
\end{array}\right]
$$

According to the Gershgorin theorem, the elements on the main diagonal of $\mathbf{S}$ are the estimated eigenvalues of $\mathbf{R}_{Y}$. Notice that most of the off-diagonal elements of $\mathbf{S}$ are zero, meaning that the Gershgorin radii $\left\{R_{i}\right\}$ are simply the absolute values of $\left\{\rho_{i}\right\}$. The Gershgorin radius $R_{m}$ is the sum of the absolute values of all $\rho_{i}$ for $i=1, \ldots m-1$.

From the standpoint of practical implementation of the Gershgorin method with transformed covariance matrix, it is not necessary to perform all the calculations given in expressions (7)-(11) to estimate the eigenvalues of $\mathbf{R}_{\mathrm{Y}}$. Instead, notice that the first $(m-1)$ elements of the main diagonal of $\mathbf{S}$ are the eigenvalues of $\mathbf{R}_{1}$ and the last element of this diagonal is the element $r_{m m}$ of the covariance matrix $\mathbf{R}_{\mathbf{Y}}$. The eigenvalues of $\mathbf{R}_{1}$ can be estimated using any method that, in this case, will process a smaller matrix, since the order of $\mathbf{R}_{1}$ is $(m-1) \times(m-1)$.

\section{NumericAl Results}

In this section, the performances of the GLRT, the MMED and the MED are assessed, under the effect of approximation errors produced by the eigenvalue estimation methods just described. Computational run-time measurements are also presented for these methods, as well as a trade-off analysis between performance and the accuracy in estimating the eigenvalues for cooperative spectrum sensing purposes.

\section{A. Performance Analysis}

Each point on the ROC curves presented hereafter was generated from 50,000 Monte Carlo events. The transmitted signal samples expressed by the elements of $\mathbf{X}$ and the elements $h_{i j}$ of the channel matrix $\mathbf{H}$ were simulated as complex Gaussian random variables with zero mean and unitary variance. The elements $h_{i j}$ were kept constant during the sensing interval, and independently generated across successive intervals. This type of channel model represents a flat and slow Rayleigh fading with unitary power gain. When a pure AWGN channel is considered, $h_{i j}=1$ for all $i$ and $j$. The noise samples in $\mathbf{V}$ were simulated by means of complex Gaussian random variables with zero mean and variance $\sigma^{2}=1 / 10^{\mathrm{SNR} / 10}$, with the SNR expressed in $\mathrm{dB}$.

Figures 1, 2 and 3 present ROC curves for the MED, the GLRT and the MMED, respectively, under different methods of eigenvalue estimation. The systemic parameters were set to $p=1$ primary transmitter, $m=6 \mathrm{CRs}$ in cooperation, $n=50$ samples collected by each $\mathrm{CR}$, signal-to-noise ratio $\mathrm{SNR}=-10 \mathrm{~dB}$ for the pure AWGN channel, $\mathrm{SNR}=-6 \mathrm{~dB}$ for the Rayleigh channel, and $K=16$ iterations in the Cholesky algorithm. This number of iterations was chosen because it has been found sufficient to practically match the spectrum sensing performance achieved when the eigenvalues 
are estimated exactly (under the inherent numerical precision) by the Matlab function eig. In these figures, the term Matlab eig is used to denote the use of the Matlab function eig; the term Gershgorin denotes the conventional Gershgorin method; and Gershgorin T denotes the Gershgorin method with transformed covariance matrix, applying the Cholesky iterations method for the estimation of the eigenvalues of $\mathbf{R}_{1}$.
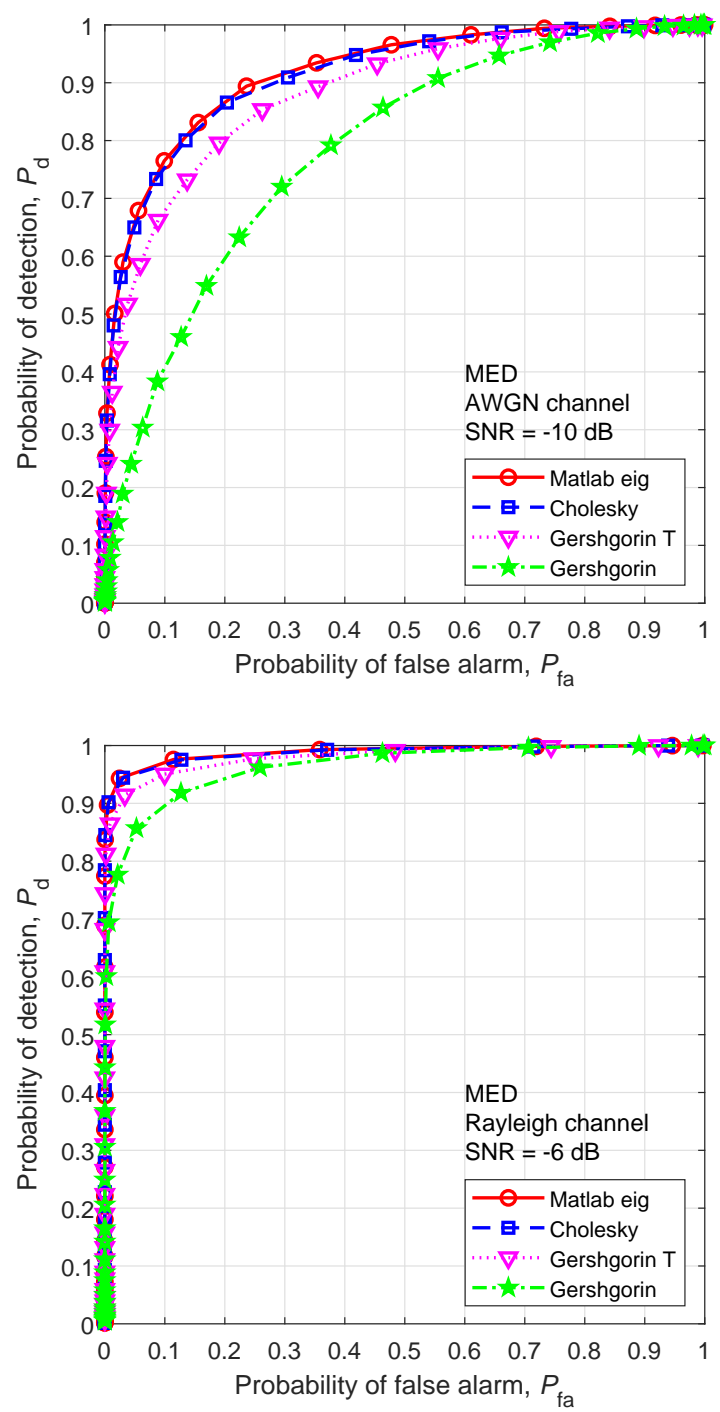

Fig. 1. Performance of the MED for $p=1, m=6, n=50$ and $K=16$ iterations in the Cholesky algorithm: AWGN channel @ SNR $=-10 \mathrm{~dB}$ (top), Rayleigh channel @ SNR $=-6 \mathrm{~dB}$ (bottom).

A first observation that can be made regarding Figures 1, 2 and 3, either when the Rayleigh or the AWGN sensing channel is concerned, refers to the performance rank of the analyzed sensing techniques, with the MED in the best position, the GLRT in the second and the MMED in the third, for the eigenvalue estimation methods using the Matlab eig function, the Cholesky iterations and the Gershgorin with transformed covariance matrix; this classification is consistent with the results reported in [6]. However, when the conventional Gershgorin method was applied, the MED achieved the best rank position, and the GLRT and the MMED exhibited approximately the same performance, with a slight, but practically negligible advantage of the MMED (except when the GLRT and the MMED performances are on the no-discrimination line, which corresponds to $P_{\mathrm{d}}=P_{\mathrm{fa}}$ ). The performance ranking is best identified by means of the areas under the ROC curves listed in Table I, where the values in boldface are the smallest for each method.
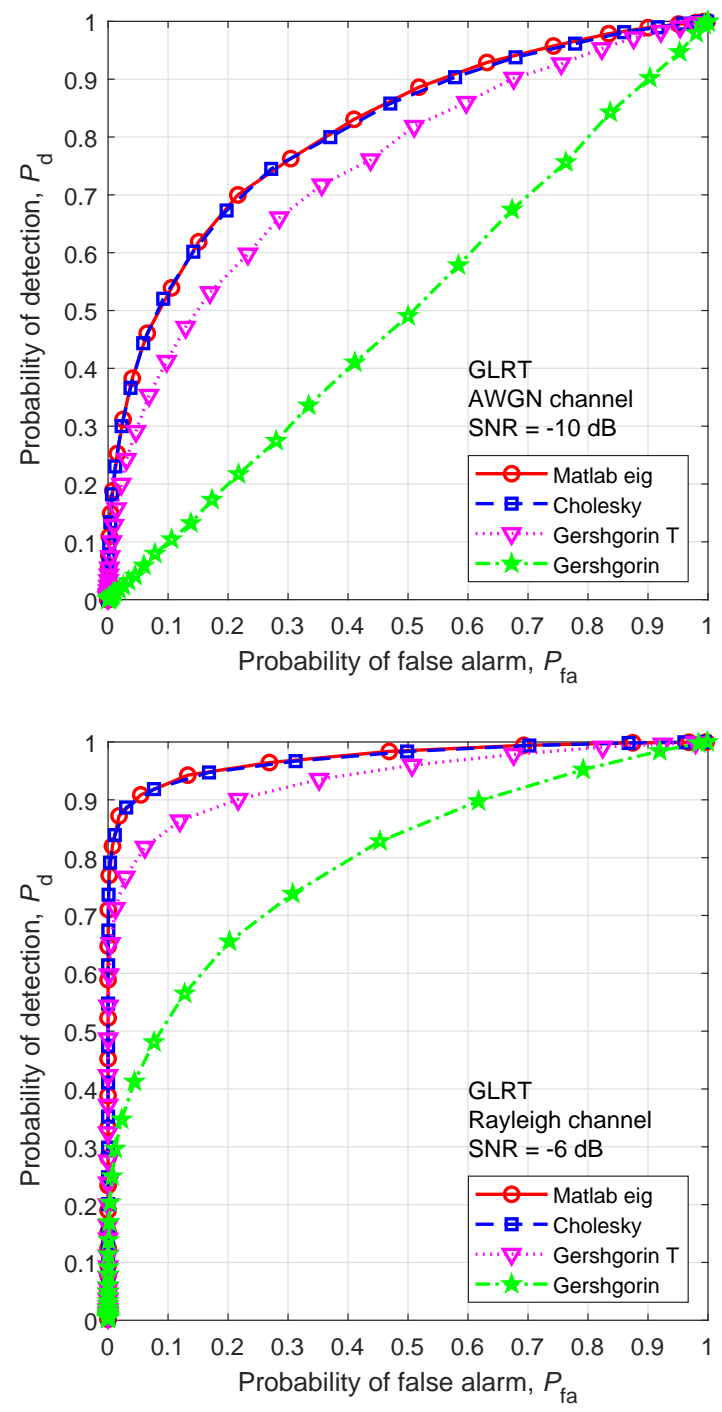

Fig. 2. Performance of the GLRT for $p=1, m=6, n=50$ and $K=16$ iterations in the Cholesky algorithm: AWGN channel @ SNR $=-10 \mathrm{~dB}$ (top), Rayleigh channel @ SNR $=-6 \mathrm{~dB}$ (bottom).

In the specific case of the AWGN sensing channel, the results shown in the graphs on the upper part of Figures 1, 2 and 3, when the Matlab eig function is used, are in close agreement with the corresponding ones in [6, Figure 1], a fact that can be used to certify the simulations used to generate the results presented in this section.

One should be aware that the SNRs applied to the AWGN and the Rayleigh fading channel are different from each other; the remaining system parameters are the same. If the SNRs were equal to one another, all performances regarding the AWGN channel would overcome the respective ones in the 
Rayleigh channel situation, as expected.

TABLE I

AREAS UNDER THE ROC CURVES SHOWN IN Figures 1, 2 AND 3. THE VALUES IN BOLDFACE ARE THE SMALLEST FOR EACH METHOD.

\begin{tabular}{|l|c|c|c|c|}
\cline { 2 - 5 } \multicolumn{1}{c|}{} & \multicolumn{4}{c|}{ Eigenvalue estimation method } \\
\hline Detector / channel & Matlab eig & Cholesky & Gershgorin T & Gershgorin \\
\hline \hline MED / AWGN & 0.9180 & 0.9133 & 0.8843 & 0.7835 \\
\hline GLRT / AWGN & 0.8126 & 0.8097 & 0.7483 & $\mathbf{0 . 5 0 0 0}$ \\
\hline MMED / AWGN & $\mathbf{0 . 7 5 3 7}$ & $\mathbf{0 . 7 5 1 8}$ & $\mathbf{0 . 7 1 8 4}$ & $\mathbf{0 . 5 0 0 0}$ \\
\hline \hline MED / Rayleigh & 0.9884 & 0.9877 & 0.9807 & 0.9638 \\
\hline GLRT / Rayleigh & 0.9721 & 0.9705 & 0.9366 & $\mathbf{0 . 7 9 4 9}$ \\
\hline MMED / Rayleigh & $\mathbf{0 . 9 4 9 3}$ & $\mathbf{0 . 9 4 7 8}$ & $\mathbf{0 . 9 2 6 8}$ & 0.8052 \\
\hline
\end{tabular}

From Figures 1, 2 and 3 it can be also noticed that the conventional Gershgorin method, which is very simple, does not provide performances as good as the ones attained by the other methods. Nonetheless, when the MED detector is applied and the target ${ }^{4}$ ROC lies around or above the pair $\left(P_{\mathrm{fa}}=0.1, P_{\mathrm{d}}=0.9\right)$, the performance achieved with conventional Gershgorin method stays not too far from the best ones. This can be observed in the graph on the bottom of Figure 1. It has been observed via other results (not presented here for conciseness) that the same behavior applies to the AWGN sensing channel. This is credited to the use of a single eigenvalue in the MED test statistic, which makes it less sensitive to eigenvalue estimation errors. An evidence that supports this argument is the similar performances of the GLRT and the MMED when the conventional Gershgorin method is applied (recall that the GLRT operates on $m$ eigenvalues, whereas the MMED operates on two). Hence, it can be concluded that the conventional Gershgorin method can be considered attractive when the MED is applied to meet standard performance metrics, like those regulated by the IEEE 802.22 standard [19].

Still referring to Figures 1, 2 and 3, it can be seen that the eigenvalue estimation via the Gershgorin theorem with transformed covariance matrix is quite attractive, since it provides performances very close to those obtained with exact estimation. However, this method requires the decomposition of $\mathbf{R}_{1}$ given in Equation (8), whose complexity may be smaller than the corresponding operation with $\mathbf{R}_{Y}$ because the order of $\mathbf{R}_{1}$ is $(m-1) \times(m-1)$ and the order of $\mathbf{R}_{\mathrm{Y}}$ is $m \times m$. Instead, referring to the end of Section III-C, one could think of using an approximate method to compute the eigenvalues of $\mathbf{R}_{1}$, for instance the Cholesky iterations, aiming at a reduced complexity at the cost of less accurate (but perhaps accurate enough) estimates. These comments are further explored in the following subsection.

\section{B. Run-time Comparison}

The effectiveness of an algorithm is commonly measured by the computational complexity of its solution, aiming at unveiling the asymptotic processing time growth as a function of the size of vector or matrix variables operated by such

\footnotetext{
${ }^{4}$ The IEEE 802.22, which is the first released standard regarding the CR technology [19], regulates that the spectrum sensing of TV signals must comply with $P_{\mathrm{fa}} \leq 0.1$ and $P_{\mathrm{d}} \geq 0.9$. If the ROC passes through the points $\left(P_{\mathrm{fa}}=0.1, P_{\mathrm{d}}=0.9\right)$, the associated AUC is around 0.96; the exact AUC depends on the specific shape of the ROC.
}
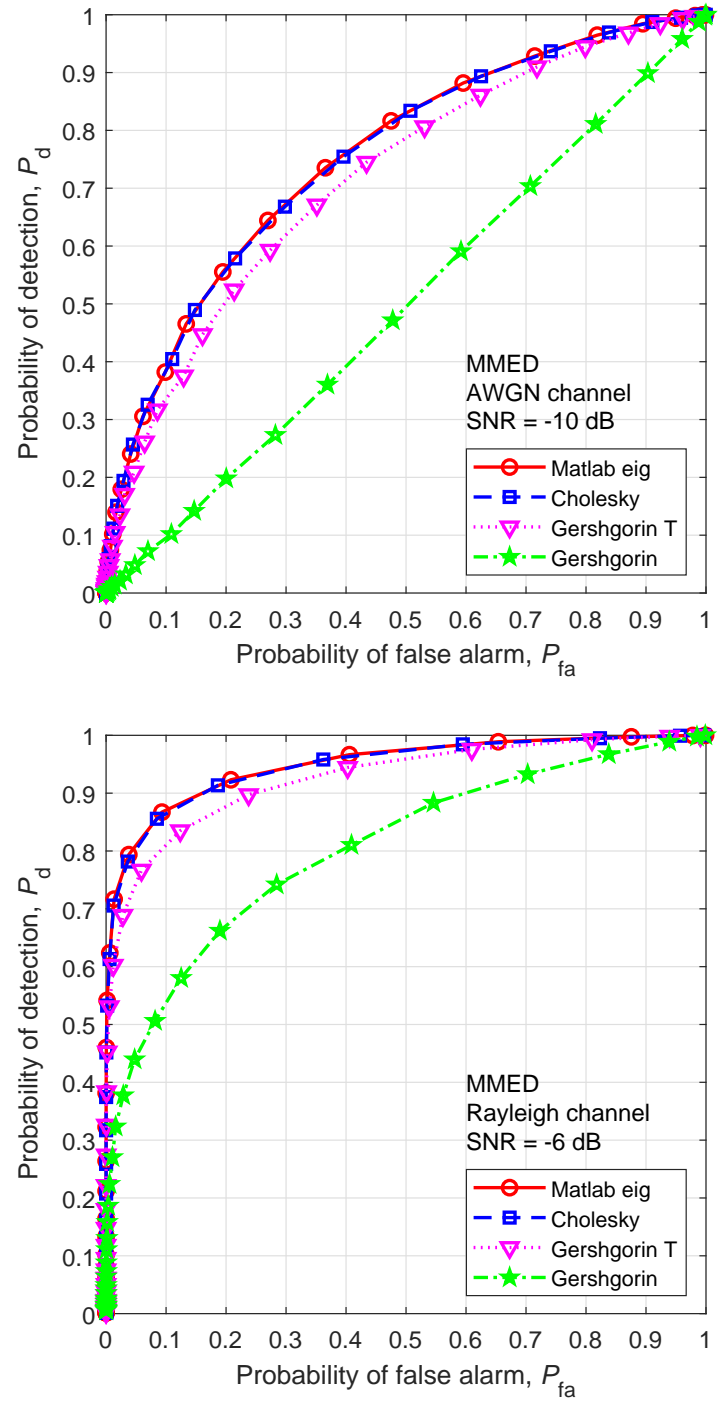

Fig. 3. Performance of the MMED for $p=1, m=6, n=50$ and $K=16$ iterations in the Cholesky algorithm: AWGN channel @ SNR $=-10 \mathrm{~dB}$ (bottom), Rayleigh channel @ SNR $=-6 \mathrm{~dB}$ (bottom).

algorithm. This processing time depends on the number of floating point operations (FLOPs) that must be performed to come up with the results.

The theoretical analysis of the computational complexity of an algorithm can be simple, such as the QR and the Cholesky decomposition [12], but the actual computational processing time will depend on the choice of the programming language, the hardware and software platforms that will execute the algorithm, the values and particularities of the parameters and variables operated, and the implementation structure of the algorithm itself.

Instead of a theoretical complexity analysis, computation run-time measurements are provided herein. The eigenvalue estimation methods were implemented via the Matlab software, which has been considered the standard tool for testing algorithms [11]. The tic and toc Matlab functions were used to measure the run-times. In order to reduce the effects of randomness, an average of 100,000 run-times of each 
eigenvalue estimation method was computed, one for each random realization of the covariance matrix $\mathbf{R}_{Y}$, under the hypotheses $\mathcal{H}_{0}$ and $\mathcal{H}_{1}$, for each value of $m$. Aiming at measuring the run-times associated only to the execution of the estimation methods, the tic command was placed just after the generation of $\mathbf{R}_{Y}$, and the toc command was placed just after the estimated eigenvalues were sorted to subsequently be used in (3), (4) or (5). The run-time of each method was measured with the other methods disabled, with the computer making no other task and disconnected from any network. The measurements were made using an HP computer with an Intel Core i7-7700 CPU @ 3.6 GHz,16 GBytes RAM, running the Matlab R2018a 64-bits, under the Windows 10 Professional.

For the system parameters, it has been chosen $p=1$ primary transmitter, $n=50$ samples per CR, SNR $=-6 \mathrm{~dB}$ over the Rayleigh sensing channel, and $K=16$ iterations in the Cholesky algorithm. The number $m$ of CRs in cooperation ${ }^{5}$, which is the order of the sample covariance matrix $\mathbf{R}_{Y}$ defined in (2), was varied from 3 to 42 in steps of 3 .

Since the absolute processing times depend on the computer hardware, run-times normalized with respect to the maximum are reported. The reference was taken as the run-time spent by the QR algorithm, which is, as already mentioned, one of the most used approaches for eigenvalue estimation. Its structure is presented in the Algorithm 2. This algorithm attains the same numerical precision of the Cholesky iterations, with half of the iterations [21]. In the Algorithm 2, the Matlab function qr has been used to compute the $\mathrm{QR}$ decomposition of the matrix $\mathbf{J}$, that is, qr $(\mathrm{J})$ returns the matrices $\mathbf{Q}$ and $\mathbf{R}$ such that $\mathbf{J}=\mathbf{Q R}$, where $\mathbf{Q}$ is an orthogonal matrix and $\mathbf{R}$ is an upper triangular matrix [10].

The results are shown in Figure 4, from where it can be seen that the processing time spent by the QR algorithm was the highest, in spite of being configured with half $(K=8)$ of the number of iterations of the Cholesky iterations method $(K=16)$. This result is consistent with the fact that the theoretical computational complexity of the QR decomposition embedded in the QR algorithm is larger than the Cholesky decomposition embedded in the Cholesky iterations method [12].

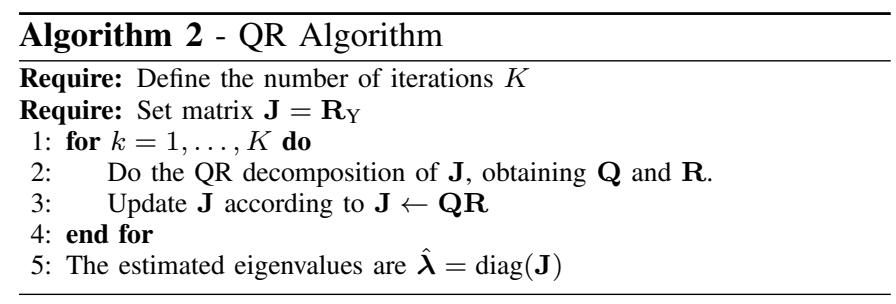

The Cholesky iterations method exhibited approximately the same run-time of the Gershgorin method with transformed matrix. It could be expected a higher run-time of the Cholesky iterations method at lower values of $m$, since it operates

\footnotetext{
${ }^{5}$ The upper limit $m=42$ for the number of CRs in cooperation has been considered enough for the purpose at hand, since in practice it is expected that this number shall not be large. In fact, $m$ should be as small as possible to save energy in the secondary network, and avoid the increase of the bandwidth of the control channel used to convey the spectrum sensing information to the fusion center [20]. Moreover, the cooperation gain acts in a diminishing-return fashion, meaning that small improvements are achieved with large $m$.
}

$\mathbf{R}_{\mathrm{Y}}$ of order $m$, whereas the Cholesky iterations used in the Gershgorin method with transformed matrix operates $\mathbf{R}_{1}$ of order $(m-1)$. The disadvantage of Cholesky iterations would be reduced with an increase in $m$, since $m$ and $(m-1)$ get close to each other. However, after $\mathbf{R}_{Y}$ is generated, the additional step of extracting $\mathbf{R}_{1}$ from $\mathbf{R}_{\mathbf{Y}}$ is implemented in the Gershgorin method with transformed matrix, penalizing its processing time and approximating the run-times of the two methods.

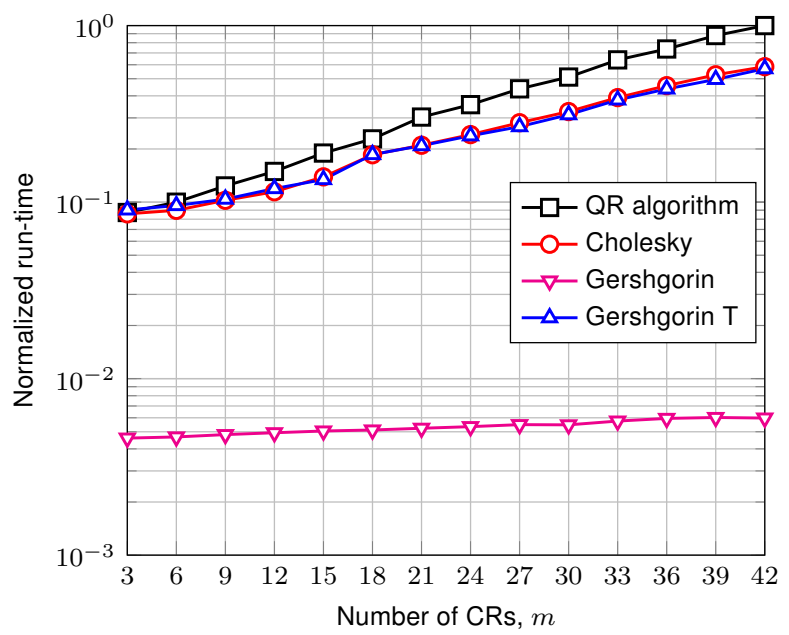

Fig. 4. Normalized run-times of the eigenvalue estimation methods.

It can also be observed from Figure 4 that the run-time of the conventional Gershgorin method increases in a very small pace as $m$ is increased, owed to the single operation of sorting the elements of the main diagonal of $\mathbf{R}_{\mathbf{Y}}$. However, one must recall that the performance of this method may be not attractive, except in situations of high SNR or in the specific case of the MED at high performance regions of the ROC curve (revisit the graph on the bottom of Figure 1).

It is worth noting that the number of iterations in the Algorithm $1(K=16)$ and in the Algorithm $2(K=8)$ were chosen as those sufficient to approximate the spectrum sensing performances provided by these algorithms to the one achieved with the exact estimation of the eigenvalues. Higher numbers of iterations increase the processing time of both, which also happens with the Gershgorin method with transformed matrix when implemented using the Cholesky iterations algorithm to estimate the eigenvalues of $\mathbf{R}_{1}$.

\section{Performance versus Eigenvalue Estimation Errors}

At this point, it becomes relevant to establish accuracy metrics of the eigenvalues estimated through the approximate solution methods with respect to the exact one, coupling such metrics with the spectrum sensing performances.

If $\lambda_{i}$ is the $i$-th exact eigenvalue computed by the Matlab eig function, and $\hat{\lambda}_{i}$ is the corresponding approximate eigenvalue estimate, the $i$-th relative error between $\lambda_{i}$ and $\hat{\lambda}_{i}$ can be defined as

$$
\varepsilon_{i}=\frac{\left|\lambda_{i}-\hat{\lambda}_{i}\right|}{\left|\lambda_{i}\right|} .
$$


Tables II and III present the average relative errors, $\bar{\varepsilon}$, and the corresponding standard deviations, $\sigma_{\bar{\varepsilon}}$, computed from sets of eigenvalue estimates made by the three methods considered herein, assuming, as before, $p=1$ primary transmitter, $m=6$ CRs, $n=50$ samples, SNR $=-6 \mathrm{~dB}$ for the Rayleigh channel, and $K=16$ iterations in the Cholesky algorithm. Each set with $m=6$ eigenvalues was obtained from 50,000 simulation runs in which the covariance matrix $\mathbf{R}_{Y}$ was generated under the hypotheses $\mathcal{H}_{0}$ (Table II) and $\mathcal{H}_{1}$ (Table III). In these tables, Cholesky denotes the Cholesky iterations method, Gershgorin refers to the conventional Gershgorin method, and Gershgorin T denotes the Gershgorin method with transformed covariance matrix, in which the Cholesky iterations method was also applied with $K=16$ iterations.

TABLE II

AVERAGE RELATIVE ERRORS, $\bar{\varepsilon}$, AND THEIR STANDARD DEVIATIONS, $\sigma_{\bar{\varepsilon}}$, FOR EIGENVALUE ESTIMATES UNDER THE $\mathcal{H}_{0}$ HYPOTHESIS.

\begin{tabular}{|c|c|c|c|c|c|c|}
\cline { 2 - 7 } \multicolumn{1}{c|}{} & \multicolumn{2}{c|}{ Cholesky } & \multicolumn{2}{c|}{ Gershgorin } & \multicolumn{2}{c|}{ Gershgorin T } \\
\hline & $\bar{\varepsilon}$ & $\sigma_{\bar{\varepsilon}}$ & $\bar{\varepsilon}$ & $\sigma_{\bar{\varepsilon}}$ & $\bar{\varepsilon}$ & $\sigma_{\bar{\varepsilon}}$ \\
\hline$\lambda_{1}$ & 0.0187 & 0.0214 & 0.2306 & 0.0565 & 0.0676 & 0.0476 \\
\hline$\lambda_{2}$ & 0.0244 & 0.0243 & 0.1390 & 0.0537 & 0.0606 & 0.0457 \\
\hline$\lambda_{3}$ & 0.0263 & 0.0258 & 0.0553 & 0.0401 & 0.0533 & 0.0433 \\
\hline$\lambda_{4}$ & 0.0269 & 0.0270 & 0.1127 & 0.0731 & 0.0763 & 0.0638 \\
\hline$\lambda_{5}$ & 0.0247 & 0.0267 & 0.2828 & 0.1083 & 0.1069 & 0.0840 \\
\hline$\lambda_{6}$ & 0.0178 & 0.0257 & 0.5234 & 0.1780 & 0.1177 & 0.0979 \\
\hline
\end{tabular}

TABLE III

AVERAGE RELATIVE ERRORS, $\bar{\varepsilon}$, AND THEIR STANDARD DEVIATIONS, $\sigma_{\bar{\varepsilon}}$, FOR EIGENVALUE ESTIMATES UNDER THE $\mathcal{H}_{1}$ HYPOTHESIS.

\begin{tabular}{|c|c|c|c|c|c|c|}
\cline { 2 - 7 } \multicolumn{1}{c|}{} & \multicolumn{2}{c|}{ Cholesky } & \multicolumn{2}{c|}{ Gershgorin } & \multicolumn{2}{c|}{ Gershgorin T } \\
\hline & $\bar{\varepsilon}$ & $\sigma_{\bar{\varepsilon}}$ & $\bar{\varepsilon}$ & $\sigma_{\bar{\varepsilon}}$ & $\bar{\varepsilon}$ & $\sigma_{\bar{\varepsilon}}$ \\
\hline$\lambda_{1}$ & 0.0027 & 0.0102 & 0.3603 & 0.0873 & 0.0972 & 0.0796 \\
\hline$\lambda_{2}$ & 0.0185 & 0.0228 & 0.1098 & 0.0931 & 0.0892 & 0.1025 \\
\hline$\lambda_{3}$ & 0.0236 & 0.0245 & 0.1255 & 0.1097 & 0.0798 & 0.0752 \\
\hline$\lambda_{4}$ & 0.0251 & 0.0262 & 0.2397 & 0.1328 & 0.1014 & 0.0850 \\
\hline$\lambda_{5}$ & 0.0235 & 0.0264 & 0.4102 & 0.1600 & 0.1180 & 0.0936 \\
\hline$\lambda_{6}$ & 0.0171 & 0.0257 & 0.6494 & 0.2312 & 0.1240 & 0.1032 \\
\hline
\end{tabular}

Tables II and III show that the average relative errors associated to the Cholesky iterations method are mostly around $\approx 0.02$, or $2 \%$. Since such method is able to yield practically the same performance achieved with the exact eigenvalues, it is concluded that estimation errors of up to $2 \%$ are not enough to affect the performances of the eigenvalue-based spectrum sensing techniques considered herein.

In the case of the Gershgorin method with transformed matrix, errors of $\approx 5 \%$ to $11 \%$ were obtained, but these values were not enough to produce a large performance variation with respect to the case in which the estimates are exact.

The conventional Gershgorin method did not achieve satisfactory performance, which is justified by the high estimation errors shown in Tables II and III, with values of $\approx 5 \%$ to $60 \%$. It is interesting to notice that, under the hypothesis $\mathcal{H}_{1}$, the estimation of the maximum eigenvalue, $\lambda_{1}$, by the Cholesky iterations method has produced an average error smaller than $\approx 0.3 \%$, meaning that if the Cholesky iterations method is coupled with the MED detector, a smaller number of iterations can be used, reducing the computational run-time.

\section{CONCLUSiOns}

This paper carried out a performance analysis of cooperative spectrum sensing techniques based on the eigenvalues of the received signal sample covariance matrix, subjected to eigenvalue estimation errors. Three different estimation methods were investigated: i) the Cholesky iterations algorithm, ii) the Gershgorin theorem with transformed covariance matrix, and iii) the conventional Gershgorin theorem. Three widely known eigenvalue-based test statistics were analyzed: i) the generalized likelihood ratio test (GLRT), ii) the maximumminimum eigenvalue detector (MMED), and iii) the maximum eigenvalue detector (MED). Simulation results have demonstrated that the Cholesky iterations and the Gershgorin theorem with transformed covariance matrix can provide satisfactory performances, while the conventional Gershgorin method is attractive only in situations of high signal-to-noise ratios, and when applied to the MED in high performance situations.

An analysis of computational run-time has been also presented, unveiling that the Cholesky iterations method is more attractive, because it is faster than the traditional QR algorithm and its accuracy is sufficiently enough to, with a few iterations, achieve a spectrum sensing performance almost identical to that obtained with the exact estimation of the eigenvalues. The Gershgorin method with transformed matrix has also shown to be attractive, because the performance penalty caused by its inherent inaccuracy is not high. In addition, the implementation complexity of the Gershgorin method with transformed matrix can be smaller than the one yielded by the Cholesky iterations method, since the factorization that is part of the former operates a matrix with a reduced-order with respect to that operated by the Cholesky iterations. However, this advantage only occurs when the order of the covariance matrix (which corresponds to the number of CRs in cooperation) is not too high, which is the typical setting in practice.

It has been also demonstrated that the test statistics are not equally sensitive to the eigenvalue estimation errors. The GLRT is the most sensitive because is operates all eigenvalues. The MMED, which operates two eigenvalues, comes subsequently in terms of robustness to estimation errors. The MED is the most robust, which is credited to the fact that it operates a single eigenvalue. However, it has been verified that the eigenvalue-based spectrum sensing is quite robust with respect to eigenvalue estimation errors, yielding practically no performance degradation for errors less than or equal to $2 \%$; little performance variations are observed for estimation errors up to around $5 \%$.

From above, it can be concluded that the present research can support the choice and implementation of eigenvaluebased spectrum sensing techniques in real hardware, since it provides the basic building blocks of suitable algorithms for solving the eigenvalue problem in a way appropriately suited to the spectrum sensing context.

As a natural deployment of the research, it is intended to implement an eigenvalue-based spectrum sensing system for digital TV signals using the universal software radio peripheral (USRP) [22] board, with the estimation of eigenvalues performed by the Cholesky iterations method. Other methods 
can also be tested, aiming at validating the results presented in this paper and obtaining new ones.

Another possible extension is the development of a model for generating eigenvalue estimation errors, thus allowing such errors to be incorporated into the performance analysis of any eigenvalue-based spectrum sensing technique, without the need of actually implementing any approximate eigenvalue estimation method. The starting point for devising such a model is the statistical analysis of the errors produced by the methods discussed herein.

The addition of quantization errors and channel errors in the approximate eigenvalues transmitted to the fusion center is also worth investigating.

\section{REFERENCES}

[1] M. A. McHenry, P. A. Tenhula, D. McCloskey, D. A. Roberson, and C. S. Hood, "Chicago spectrum occupancy measurements \& analysis and a long-term studies proposal," in Proc. of the First International Workshop on Technology and Policy for Accessing Spectrum, Boston, Massachusetts, USA, 2006, doi: 10.1145/1234388.1234389.

[2] S. Haykin, "Cognitive radio: brain-empowered wireless communications," IEEE Journal on Selected Areas in Communications, vol. 23, no. 2, pp. 201-220, February 2005, doi: 10.1109/JSAC.2004.839380.

[3] I. F. Akyildiz, B. F. Lo, and R. Balakrishnan, "Cooperative spectrum sensing in cognitive radio networks: A survey," Physical Communication, vol. 4, no. 1, pp. 40 - 62, 2011, doi: 10.1016/j.phycom.2010.12.003.

[4] Y. Arjoune and N. Kaabouch, "A comprehensive survey on spectrum sensing in cognitive radio networks: Recent advances, new challenges, and future research directions," Sensors, vol. 19, no. 1, 2019, doi: 10.3390/s19010126. [Online]. Available: http://www.mdpi.com/1424$8220 / 19 / 1 / 126$

[5] Y. Zeng, Y.-C. Liang, A. T. Hoang, and R. Zhang, "A review on spectrum sensing for cognitive radio: Challenges and solutions," EURASIP Journal on Advances in Signal Processing, vol. 2010, no. 1, p. 381465, Jan 2010. [Online]. Available: https://doi.org/10.1155/2010/381465

[6] B. Nadler, F. Penna, and R. Garello, "Performance of eigenvalue-based signal detectors with known and unknown noise level," in Proc. of the IEEE International Conference on Communications, Kyoto, Japan, June 2011, pp. 1-5, doi: 10.1109/icc.2011.5963473.

[7] D. A. Guimarães, "Robust test statistic for cooperative spectrum sensing based on the Gerschgorin circle theorem," IEEE Access, vol. 6, pp. 2445-2456, December 2018, doi: 10.1109/ACCESS.2017.2783443.

[8] R. L. Dykstra, "Establishing the positive definiteness of the sample covariance matrix," Ann. Math. Statist., vol. 41, no. 6, pp. 2153-2154, 12 1970. [Online]. Available: https://doi.org/10.1214/aoms/1177696719

[9] A. v. d. Bos, Parameter Estimation for Scientists and Engineers. New York, NY, USA: Wiley-Interscience, 2007.

[10] G. Strang, Introduction to Linear Algebra. $5^{\text {th }}$ ed., Wellesley-Cambridge Press, 2016. [Online]. Available: https://www.amazon.com/IntroductionLinear-Algebra-Gilbert-Strang/dp/0980232775

[11] G. H. Golub and H. A. van der Vorst, "Eigenvalue computation in the 20th century," Journal of Computational and Applied Mathematics, vol. 123 , no. 1, pp. 35 - 65, November 2000, doi: 10.1016/S03770427(00)00413-1.

[12] J. Demmel, Applied Numerical Linear Algebra. Philadelphia, PA, USA: Society for Industrial and Applied Mathematics, 1997. [Online]. Available: https://www.amazon.com.br/Applied-NumericalLinear-Algebra-Demmel/dp/0898713897

[13] D. Kressner, Numerical Methods for General and Structured Eigenvalue Problems. Berlin, Heidelberg, Germany: Springer, 2006. [Online]. Available: https://books.google.com.br/books?id=j7kTw0wP8eIC

[14] I. Bravo, C. Vazquez, A. Gardel, J. Lazaro, and E. Palomar, "High level synthesis FPGA implementation of the Jacobi algorithm to solve the eigen problem," Mathematical Problems in Engineering, vol. 2015, pp. 1-11, March 2015, doi: 10.1155/2015/870569.

[15] A. Lopez-Parrado and J. Velasco-Medina, "Efficient systolic architecture for Hermitian eigenvalue problem," in Proc. of the IEEE Colombian Workshop on Circuits and Systems, Barranquilla, Colombia, November 2012, pp. 1-6, doi: 10.1109/CWCAS.2012.6404064.

[16] J. H. Wilkinson, C. Reinsch, and F. L. Bauer, Handbook for Automatic Computation, Linear Algebra. Springer Berlin Heidelberg, 1986, doi: $10.1137 / 1014116$.
[17] A. Krishnamoorthy and K. Kocagoez, "Singular values using cholesky decomposition," CoRR, February 2012. [Online]. Available: https://arxiv.org/pdf/1202.1490

[18] Hsien-Tsai Wu, Jar-Ferr Yang, and Fwu-Kuen Chen, "Source number estimators using transformed Gerschgorin radii," IEEE Transactions on Signal Processing, vol. 43, no. 6, pp. 1325-1333, June 1995, doi: 10.1109/78.388844.

[19] The Institute of Electrical and Electronic Engineers, IEEE. (2011) IEEE 802 Part 22: Cognitive Wireless RAN Medium Access Control (MAC) and Physical Layer (PHY) Specifications: Policies and Procedures for Operation in the TV Bands. [Online]. Available: http://standards.ieee.org/getieee802/download/802.22-2011.pdf

[20] C. H. Lim, "Resource-efficient transmission for report channel in cooperative spectrum sensing," Electronics Letters, vol. 50, no. 16, pp. 1171-1173, July 2014.

[21] K. Vince Fernando and B. N. Parlett, "Implicit Cholesky algorithms for singular values and vectors of triangular matrices," Numerical Linear Algebra with Applications, vol. 2, no. 6, pp. 507-531, 1995, doi: 10.1002/nla.1680020604

[22] Ettus Research (a National Instruments brand). Universal software radio peripheral product selector homepage. Accessed: 2019-06-06. [Online]. Available: https://www.ettus.com/usrp-product-selector

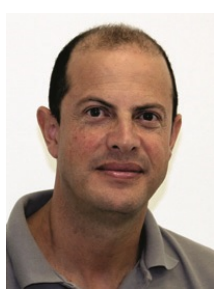

Dayan Adionel Guimarães has a Ph.D. in Electrical Engineering from State University of Campinas, UNICAMP, Brazil. He is a Senior Lecturer and a Researcher with the National Institute of Telecommunications, INATEL. His research interests are fixed and mobile wireless communications, radio propagation, simulation of digital communication systems, spectrum sensing for cognitive radio, and applied convex optimization and signal processing.

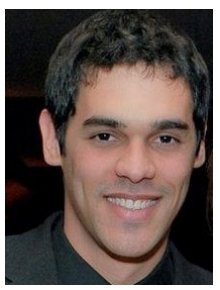

Guilherme Pedro Aquino has a Ph.D. in Electrical Engineering from the Federal University of Itajubá, UNIFEI. Currently, he is with the National Institute of Telecommunications, INATEL, as a professor and researcher at the Reference Center for Radio-communications, conducting researches in non-orthogonal multiple access and efficient cooperative spectrum sensing strategies.

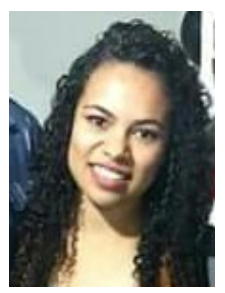

Ana Carolina Vivian dos Reis has a Bachelor in Telecommunications Engineering from the National Institute of Telecommunications, INATEL, and is an Electronics Technician from the Francisco Moreira da Costa Technical School of Electronics, ETE. She has interest in optical communications, cellular systems, cognitive radio systems, antennas and digital transmission.

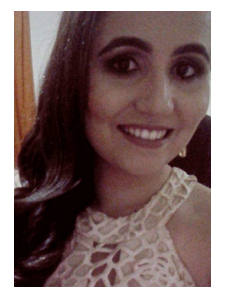

Rafaela Rosa Santos has a Bachelor in Telecommunications Engineering from the National Institute of Telecommunications, INATEL, and is an Electronics Technician from the Francisco Moreira da Costa Technical School of Electronics, ETE. She has interest in Internet of Things, spectrum sensing for cognitive radio applications, mobile communications, optical communications and embedded systems. 